\title{
Detection of Portal Venous Gas by Ultrasonography after Liver Transplantation: A Possible Early Sign of Bacterial Translocation
}

\author{
Takanobu Shigeta $^{1}$, Seisuke Sakamoto ${ }^{1}$, Shunsuke Nosaka ${ }^{2}$, Akinari Fukuda ${ }^{1}$, Hiroyuki Kanazawa ${ }^{1}$, \\ Hajime Uchida ${ }^{1}$, Ikumi Hamano ${ }^{1}$, Mureo Kasahara ${ }^{1}$ \\ ${ }^{1}$ Division of Transplant Surgery, National Center for Child Health and Development, Tokyo, Japan \\ ${ }^{2}$ Division of Radiology, National Center for Child Health and Development, Tokyo, Japan \\ Email: shigeta-t@ncchd.go.jp
}

Received May 1, 2012; revised June 3, 2012; accepted June 18, 2012

\begin{abstract}
This portal venous gas (PVG) is usually associated with intra-abdominal catastrophe. However, there are some reports regarding benign causes of PVG, including the cases related to organ transplantation. We present 3 cases with PVG undergoing living donor liver transplantation (LDLT). All cases simultaneously demonstrated intra-abdominal complications and revealed a bacterial blood culture. We successfully managed all cases with immediate intervention for the possible occurrence of bacterial translocation. Our experience indicates that the detection of PVG by USG is an alarming finding, as a possible early sign of bacterial translocation after liver transplantation. When PVG is detected by USG, it is important to pay prompt attention to bacterial translocation.
\end{abstract}

Keywords: Liver Transplantation; Portal Venous Gas; Bacterial Translocation

\section{Introduction}

Portal venous gas (PVG) has been reported in association with intra-abdominal events that include intestinal ischemia, perforation, obstruction, or an intra-abdominal abscess. Patients with PVG generally have a poor survival rate of less than $25 \%$ due to the severity of the underlying pathologic condition that is usually intestinal necrosis [1]. On the other hand, PVG has been detected in cases following medical procedures inducing iatrogenic gastric and bowel dilatation, such as colonoscopy, and their prognosis is favorable and a laparotomy is not required [2]. In the setting of organ transplantation, especially the liver, PVG has been reported in association with venovenous bypass, the administration of OKT3 [3], and Epstein-Barr virus (EBV)-associated post-transplant lymphoproliferative disease (PTLD) [4] other than those causes described above. Chezmar, et al. previously described that PVG is a common finding in the first 2 weeks after hepatic transplantation and may not have grave prognostic significance [5]. This center had performed 190 pediatric living donor liver transplantations (LDLT) by the end of March 2012. Among them, 3 patients (1.6\%) showed PVG after LDLT (Table 1). All cases simultaneously demonstrated intra-abdominal complications, which might be associated with the appearance of PVG. This report presents 3 cases with the detection of PVG by ultrasonography (USG) after LDLT, who was successfully treated and the possible relationship between PVG and bacterial translocation after liver transplantation is discussed.

Table 1. The details of the cases with the detection of portal venous gas after LDLT.

\begin{tabular}{|c|c|c|c|c|c|c|c|c|}
\hline $\begin{array}{c}\text { Case } \\
\text { No. }\end{array}$ & Age/Gender & $\begin{array}{c}\text { Body weight } \\
\text { (kg) }\end{array}$ & $\begin{array}{l}\text { Original } \\
\text { disease }\end{array}$ & $\begin{array}{l}\text { Graft type/ } \\
\text { GRWR (\%) }\end{array}$ & PVG onset & $\begin{array}{l}\text { Intestinal } \\
\text { complication }\end{array}$ & Blood culture & Management/Outcome \\
\hline 1 & $4 \mathrm{~m} / \mathrm{M}$ & 5.495 & BA & reduced LLS/2.64\% & POD 8 & Perforation & Enterobactor cloacae & Surgery Antibiotics/Alive \\
\hline 2 & $5 \mathrm{~m} / \mathrm{M}$ & 9.4 & OTCD & reduced LLS/2.83\% & POD 7 & Bowel dilation & Staphylococcus xylosus & D/C nutrition Antibiotic/Alive \\
\hline 3 & 3 у $10 \mathrm{~m} / \mathrm{F}$ & 16.9 & GSD Ib & LLS/1.39\% & POD 33 & Melena & Enterobacter cloacae & D/C nutrition Antibiotics/Alive \\
\hline
\end{tabular}

BA: Biliary atresia; D/C: Discontinue; GRWR: Graft-to-recipient body weight ratio; GSD: Glycogen storage disease; LDLT: Living donor liver transplantation; LLS: Left lateral segment; OTCD: Ornithine transcarbamylase deficiency; POD: Postoperative days; PVG: Portal venous gas. 


\section{Case Report}

\subsection{Case 1}

A 4-month-old male with biliary atresia underwent LDLT using a reduced left lateral segment graft from his mother. He stayed in the intensive care unit for systemic management before undergoing LDLT. On postoperative day (POD) 4, he became febrile and a bacterial blood culture simultaneously revealed infection with Enterobacter cloacae. Antibiotics were immediately administered, however, PVG was detected by USG on POD 8 , and an infectious origin in the abdominal cavity was suspected. The perative findings showed small bowel perforation. No PVG was detected with USG after surgery. Antibiotics were administered for two weeks and the patient's infectious status improved. He was discharged two months after undergoing LDLT.

\subsection{Case 2}

A 2-year-and-9-month-old male with ornithine transcarbamylase deficiency underwent LDLT using a reduced left lateral segment graft from his father. He stayed in the intensive care unit due to intractable hyperammonemia before undergoing LDLT. Following LDLT, bowel dilatation gradually became prominent and the patient became febrile. USG detected PVG on POD 7. Enteral feeding was immediately stopped and antibiotics were administered due to a suspected diagnosis of bacterial translocation due to paralytic ileus. On POD 9, a bacterial blood culture detected Staphylococcus xylosus. USG performed on the following day failed to detect any PVG. The administration of antibiotics continued for two weeks and the patient became afebrile. He was discharged three months after undergoing LDLT.

\subsection{Case 3}

A 4-year-old female with glycogen storage disease type Ib underwent LDLT for repeated episodes of hypoglycemia and infection due to neutropenia. She received a left lateral segmental graft from her 35-year-old father. Anti-CD20 monoclonal antibody $\left(375 \mathrm{mg} / \mathrm{m}^{2}\right.$ body surface area) was administered and plasmapheresis was performed three times before her LDLT because the blood type was incompatible between the recipient and the donor. The immunosuppression regimen after LDLT consisted of tacrolimus, steroids, and mycofenolate mofetil. Her liver function tests (LFTs) gradually worsened from the second week after LDLT, and steroid pulse therapy was initiated due to the diagnosis of acute cellular rejection. She had an episode of melena on POD 23, and endoscopic examinations revealed no obvious source of gastrointestinal bleeding. The gastrointestinal bleeding had been spontaneously stopped, and enteral feeding was resumed. USG suddenly detected PVG one week later (Figure 1). Simultaneously, she became febrile, and a bacterial blood culture detected the pathogenic bacterium of intestinal origin (Enterobacter cloacae), which possibly indicated bacterial translocation. Enteral feeding was immediately stopped, and antibiotics were started. In addition, the immunosuppression was withdrawn. No PVG was detected by USG on the following day. The administration of antibiotics was completed after two weeks, and she became afebrile. Afterwards, she demonstrated a good recovery, and she was discharged three months after undergoing LDLT.

\section{Discussion}

Most cases of PVG are associated with intra-abdominal events. However, its pathogenesis is not fully understood. PVG, which indicates the translocation of air into the portal vein system, requires the loss of bowel wall mucosa
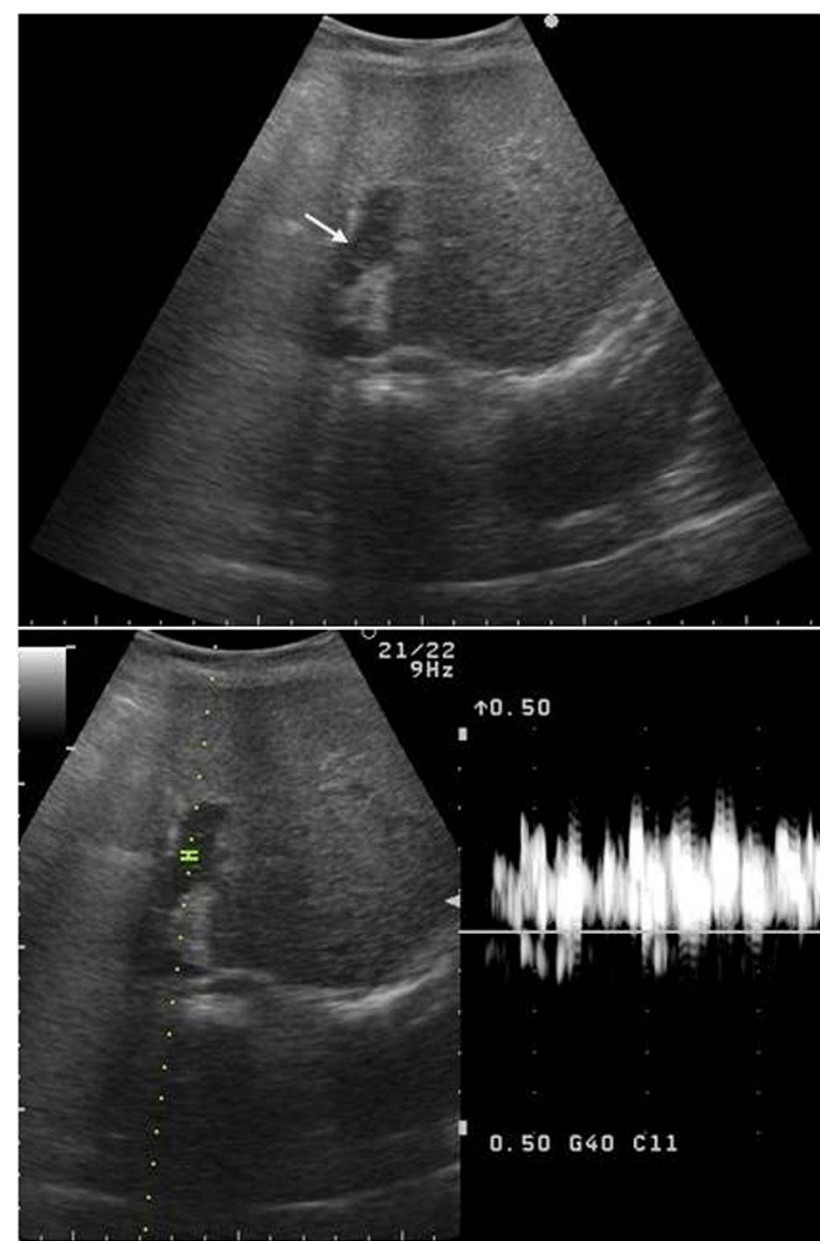

Figure 1. The ultrasound findings at the time of the appearance of PVG. Fast drifting highly echogenic particles (arrow) within the portal vein lumen were visible in the B-mode, and the Doppler mode showed intermittent high amplitude sinals. 
integrity [4]. Sebastià, et al. described the causes of the loss of bowel wall mucosa integrity and divided them into four groups: 1) Intestinal wall alterations (inflammatory bowel disease, mesenteric ischemia); 2) Bowel distention (gastric and bowel dilatation due to spontaneous, traumatic, and iatrogenic causes); 3) Intra-abdominal sepsis (diverticulitis, abdominal wall gangrene, pylephlebitis); 4) Unknown causes [2]. In their series, "organ transplantation" was indefinitely listed as one of the unknown causes. Furthermore, they quoted from a paper from Chezmar, et al. [5], who concluded that PVG was a common finding in the first 2 weeks after hepatic transplantation and may not have grave prognostic significance. However, the details of their paper noted PVG to be associated with sepsis, ileus, graft failure, and clostridium colonization in four out of 7 patients, which means that the appearance of PVG is an alarming finding for an immunocompromised host after liver transplantation.

In our series, the patient in Case 1 experienced the severest complications. Although a bacterial blood culture was assayed in Case 1, we were unable to identify the infectious origin at that time. However, we performed laparotomy after detecting PVG on POD 8, and the patient successfully treated after surgery. In the other two cases, we were able to prevent the symptoms from worsening because treatment, including the discontinuation of enteral feeding and the administration of antibiotics, was initiated as soon as PVG was detected by USG. Although PVG is not always detected in all patients with abdominal complications, it is important to recognize PVG as a possible early sign of bacterial translocation and to initiate treatment promptly.

Portal venous gas can be easily detected by USG, but it can also be missed, if the characteristics of the findings of PVG are not recognized. Fast drifting highly echogenic particles within the portal vein lumen are visible in the B-mode, and the Doppler mode shows intermittent high amplitude signals and a broadened range of velocities at any given point in time are indicative of PVG. The acoustic amplified signal displays a characteristics sound [6]. Computed tomography (CT), which can demonstrate PVG more accurately and detect the presence of pneumatosis intestinalis, should be performed, when USG shows PVG. Both of the radiologic modalities allow an earlier detection of small quantities of gas in the portal tract [2]. USG might be more sensitive for identifying PVG at an early stage than CT, because CT did not show any gas that would suggest PVG or pneumatosis intestinalis in the current series. In addition, USG has an advantage due to its portability, especially for severely ill patients.

The three patients showing PVG after LDLT in the current series had simultaneous intra-abdominal compli- cations. Furthermore, all of them showed positive bacterial culture of blood, and pathogenic bacteria of intestinal origin were detected in two patients. All of these patients were successfully managed with immediate intervention for the possible occurrence of bacterial translocation. Bacterial translocation is defined as the phenomenon by which live bacteria, their products, or both cross the intestinal barrier. Three mechanisms are thought to promote bacterial translocation: small bowel bacterial overgrowth, immune deficiency, and physical damage to the intestinal mucosa and vasculature that causes increased permeability [7]. It is easy to assume that liver transplant recipients might be vulnerable to bacterial translocation due to their immunocompromised state. Moreover, each of the patients had risk factors for infection. The patient in this report had glycogen storage disease type Ib, which shows both neutropenia and neutrophil dysfunction. In addition, strengthening the immunosuppression with the pretransplant administration of anti-CD20 monoclonal antibody for the ABO-incompatible LDLT or steroid pulse therapy before the appearance of PVG might have had a negative impact on the occurrence of bacterial translocation. Both of the other patients were not only infants, but also managed in the intensive care unit before LDLT due to severe illness. The administration of agents that enhance the immune function, such as probiotics should be considered in liver transplant recipients with a critically impaired host immunity to prevent the occurrence of bacterial translocation [8].

In summary, the detection of PVG by USG is an alarming finding since it is a possible early sign of bacterial translocation after liver transplantation. When PVG is detected by USG, it is therefore important to promptly treat bacterial translocation.

\section{REFERENCES}

[1] P. R. Liebman, M. T. Pattern, J. R. Benfield, et al., "Hepaticportal Venous Gas in Adults: Etiology, Pathophysiology, and Clinical Significance," Annals of Surgery, Vol. 187, No. 3, 1978, pp. 281-287. doi:10.1097/00000658-197803000-00012

[2] C. Sebastià, S. Quiroga, E. Espin, et al., "Portomesenteric Vein Gas: Pathologic Mechanisms, CT Findings, and Prognosis," Radiographics, Vol. 20, No. 5, 2000, pp. 1213-1224.

[3] J. P. Waymack, I. Penn, M. R. First, et al., "Portal Vein Gas and Sepsis after Administration of OKT3,” Lancet, Vol. 329, No. 8539, 1987, p. 984. doi:10.1016/S0140-6736(87)90338-2

[4] M. A. Wallot, J. Klepper, P. Clapuyt, et al., "Repeated Detection of Gas in the Portal Vein after Liver Transplantation: A Sign of EBV-Associated Post-Transplant Lymphoproliferation?” Pediatric Transplantation, Vol. 6, 
No. 4, 2002, pp. 332-336.

doi:10.1034/j.1399-3046.2002.02001.x

[5] J. L. Chezmar, R. C. Nelson and M. E. Bernardino, "Portal Venous Gas after Hepatic Transplantation: Sonographic Detection and Clinical Significance,” American Journal of Roentgenology, Vol. 153, No. 6, 1989, pp. 12031205.

[6] M. Lafortune, B. C. Trinh, P. N. Burns, et al., "Air in the Portal Vein: Sonographic and Doppler Manifestations,”
Radiology, Vol. 180, No. 3, 1991, pp. 667-670.

[7] S. M. Lichtman, "Bacterial Translocation in Humans," Journal of Pediatric Gastroenterology and Nutrition, Vol. 33, No. 1, 2001, pp. 1-10. doi:10.1097/00005176-200107000-00001

[8] K. Madsen, "Probiotics in Critically Ill Patients,” Journal of Clinical Gastroenterology, Vol. 42, 2008, pp. S116S118. doi:10.1097/MCG.0b013e31817283cb 